\title{
Randomized Assessor-Blinded Controlled Trial on the Efficacy and Safety of Virgin Coconut Oil versus Mineral Oil as a Therapeutic Moisturizer for Senile Xerosis
}

\author{
Mariecon O. Escuadro-Chin, Michael Marc C. Maaño and Belen L. Dofitas \\ Section of Dermatology, Department of Medicine, College of Medicine and Philippine General Hospital, University of the Philippines Manila
}

\begin{abstract}
Background. Xerosis is one of the most common dermatologic complaints in the elderly. People in the tropics, including the Philippines, have effectively used coconut oil as a traditional moisturizer for centuries. Its film-forming qualities allow it to act as a skin moisturizer and as a protectant against moisture loss. To date, only one randomized clinical trial has proven the efficacy and safety of coconut oil as a skin moisturizer for xerosis. With the increasing number of VCO products in the market, this study aimed to validate the use of an indigenous agricultural product, virgin coconut oil, for senile xerosis. With its moisturizing, antioxidant, and antiseptic effects, VCO may be superior and more cost-effective compared to the frequently used synthetic mineral oil.
\end{abstract}

Objective. This study aimed to determine the efficacy and safety of virgin coconut oil compared to mineral oil for the treatment of senile xerosis.

Methods. This was a community-based assessor-blinded, randomized controlled trial, which included elderly patients with mild to moderate senile xerosis of the legs. Participants were instructed to apply the test oil twice daily to the legs for 2 weeks. The following primary outcomes were measured at baseline and 2-weeks post treatment: skin dryness (over-all dry skin score, ODSS), skin hydration (corneometer readings), skin lipid content (sebumeter readings), and quality of life (Dermatology Life Quality Index). Secondary outcomes like patient-assessed clinical efficacy and adverse effects were also measured.

Oral Presentation and Poster Exhibit: Maria Duran Symposium/ Maria Duran Fellowship Award - $3^{\text {rd }}$ Continental Congress of Dermatology, October 24, 2012, Durban, South Africa.

Poster presented at the American Contact Dermatitis Society Annual Meeting, February 2013, Miami Florida, USA.

Abstract cited in Dermatitis Journal, Volume 24 Issue no. 42013 Annual ACDS Meeting Abstracts, January 2013.

$1^{\text {st }}$ Place - Philippine Dermatological Society Research Forum (Original Studies Category), November 2010, Mandaluyong City, Metro Manila, Philippines.

$2^{\text {nd }}$ Place - Department of Medicine Annual Research Forum (Original Studies Category), 2009, Philippine General Hospital, Manila, Philippines

Corresponding author: Mariecon O. Escuadro-Chin, MD, FPDS Section of Dermatology

Department of Medicine

Philippine General Hospital

University of the Philippines Manila

Taft Avenue, Manila 1000, Philippines

Email:con_md@yahoo.com
Results. A total of 148 participants ( 59 males, 89 females) with mean age of 68 years (SD 6.02) were included in the study. Eighty-one (81) were assigned under the VCO group and 67 under the mineral oil group. There was a total of 25 dropouts, 7 in the virgin coconut oil group, and 18 in the mineral oil group. The distribution of the patients' ODSS after treatment with VCO and mineral oil showed a trend towards improvement: $43 \%$ in the VCO group had no visible signs of leg xerosis versus $22.4 \%$ in the mineral oil group. The proportion of participants with $>1$ point decrease in ODSS, was statistically greater in the VCO group at 74\% (60/81) compared to the mineral oil group, 34\% (23/67) $(p<0.0001)$. VCO showed significantly greater skin hydration at $74 \%(60 / 81)$ as compared to $46 \%(31 / 67)$ in the mineral oil group $(p<0.0010)$. Improvement in the skin lipid content using the sebumeter showed $82.7 \%$ $(67 / 81)$ in the VCO group compared to $61.2 \%(41 / 67)$ in the mineral oil group $(p=0.6591)$. Moreover, the patients' perceived efficacy of the oil applied on their skin was $29.6 \%(24 / 81)$ in the VCO group compared 
to $5.9 \%(4 / 67)$ in the mineral oil group $(p=0.0030)$. Baseline DLQI scores showed no significant difference in the assessed quality of life of the patients between the two treatments $(p=0.0161)$.

Over all, the VCO group showed $32.1 \%(26 / 81)$ treatment success compared to $8.9 \%(6 / 67)$ in the mineral oil group ( $p=0.004614)$. Adverse events in the 2 groups were all mild and transient with $8 \%(6 / 74)$ patients in the VCO group and $26.5 \%(13 / 49)$ in the mineral oil group $(p=0.089)$.

Conclusion. Among elderly patients with mild to moderate xerosis, 2-week topical application of VCO was superior to mineral oil in the immediate improvement of leg xerosis based on primary outcome measures of the Over-all Dry Skin Score (ODSS), corneometer, sebumeter readings, and the Dermatology Life Quality Index.

Key Words: senile xerosis, coconut oil, mineral oil

\section{INTRODUCTION}

The study of diseases that impact the elderly population is a crucial and growing area of interest in medicine. However, skin problems in the elderly population have rarely been the topic of scientific research. ${ }^{1}$

Xerosis is one of the most common dermatologic complaints in the elderly. It is due to a decrease both in the lipids of the stratum corneum and in the activity of sebaceous and sweat glands with advancing age. Once xerosis sets in, the vicious cycle of flaking, fissuring, inflammation, dermatitis, and infection develop. This needs to be broken to disable the process and prevent complications. Liberal use of moisturizers reduces scaling by enhancing the corneo-desmosome degradation process. Significantly, oils have the capacity to improve epidermal barrier function and help prevent transepidermal water loss. A moisturizer with antiseptic, antibacterial and anti-oxidant effects is recommended for senile xerosis. ${ }^{2-3}$

People in the tropics have effectively used various kinds of oil as a traditional moisturizer for centuries. Among the commonly used ones are mineral oil and coconut oil. Mineral oil is distilled from petroleum, the very same inorganic underground product that is refined to become gasoline for engines. It is also frequently used as an occlusive agent and is a common ingredient among the common moisturizers. Occlusives coat the stratum corneum to inhibit transepidermal water loss, yield an emollient effect that is well suited to smoothen and soothe dry skin. Coconut oil on the other hand is made of fatty acids that are a normal component of the skin barrier, including stable, saturated, medium chain fatty acids - lauric, capric, and caprylic - and the longer chain myristic acid. All four fatty acids serve to replace lost lipids in the skin barrier. Its film-forming quality allows it to moisturize the skin and protect against water loss. ${ }^{4}$ In addition, it is also antiseptic and an antioxidant..$^{5-7}$

When applied topically, coconut oil has a very low risk of allergic reaction and pruritus. ${ }^{8,9}$

This study aimed to determine the effectiveness of VCO compared to mineral oil in the treatment of senile xerosis.

\section{OBJECTIVES}

The general objective of this study was to assess the efficacy and safety of VCO compared to mineral oil as a therapeutic moisturizer for mild to moderate senile xerosis.

The specific objectives were to compare VCO and mineral oil change in (1) the over-all dry skin score (ODSS), (2) quality of life scores (QOL), (3) skin hydration using a Corneometer, (4) skin lipids using a Sebumeter; and (5) adverse effects.

\section{MATERIALS AND METHODS}

\section{Setting and Patients}

We recruited senior citizens from three rural barangays in General Trias, Cavite through purposive sampling. Inclusion criteria were: patients aged 60 years and above, either sex, and with mild to moderate xerosis of the legs. Measurements of the ODSS, corneometer, and sebumeter readings were taken on the anterior aspect of both legs immediately below the tibial tuberosity. Participants were considered to have mild to moderate xerosis if their Over-all Dry Skin Score ranged from 1 to 3, characterized as 1, the presence of only fine scales, roughness and dullness; 2, small to few large scales, with roughness and whitish appearance, and 3, with scaling, advanced roughness, redness, eczematous changes, and cracks. Complete dermatological examination was conducted.

Patients with known hypersensitivity to the test oils; those with open wounds, abrasions, erosions, and ulcers on the test site; and paraplegics or patients with altered sensation on the test sites were excluded from the study.

The study was conducted in accordance with the Helsinki Declaration. The Expanded Hospital Research Office of the Philippine General Hospital approved the study protocol. All study participants gave written informed consent.

\section{Sample Size Calculation}

We determined that, with an $\alpha$ of $0.05, \beta$ of 0.20 (power $80 \%)$, and a distinguishable difference $(\Delta)$ in proportion of patients of $30 \%$ (ex. $90 \%$ for one treatment and $60 \%$ for the other), we would need 120 participants, 60 per treatment group. To allow for missing data and loss to follow-up, we aimed to recruit 72 patients per group, or 144 in total. 
Efficacy and Safety of Virgin Coconut Oil for Senile Xerosis

\section{Intervention and Control}

$\mathrm{VCO}$ is an edible vegetable oil that is extracted from fresh coconut meat and is processed using only physical and natural means. It is colorless at or above $30^{\circ} \mathrm{C}$ and turns white in its solid form but with a characteristic aroma. The oil contains less than $1 \%$ moisture, and consists of $92 \%$ saturated fatty acids and $56.4 \%$ lauric acid. The VCO was acquired from Peter Paul Company (Candelaria, Quezon). The company laboratory ensured complete physicochemical and microbiological testing for each production. ${ }^{10}$

Mineral oil, on the other hand, is transparent, colorless, odorless oil related to white petrolatum, composed mainly of alkanes and cyclic paraffins. The mineral oil was sourced from MCB Drug, Manila.

\section{Randomization, treatment allocation, and blinding}

Participants were randomized using a computergenerated list in balanced blocks of 10 . Treatment allocation was done by numbered, sealed opaque envelopes issued in batches of 10. A third party placed the VCO and mineral oil in identical white plastic bottles under a centralized preparation. It was impossible to completely blind the study participants to the treatment due to the distinct smell of VCO.

These bottles were sequentially numbered and arranged according to the allocation list. The treatment codes were kept in a sealed envelope by the secondary investigator. The code was broken only after the final analysis had been completed. If a serious adverse event occurred in a patient, then the code of this patient would have been revealed. The primary investigator was blinded as to which intervention each participant received.

\section{Study procedure}

We instructed the participants to apply the assigned test oil twice a day (i.e., after bath and at bedtime) on both legs, for two consecutive weeks. Experimental doses for the study used the Finger Tip Unit (FTU) method. They were instructed to apply 8 fingertip units (FTU) per leg. An instructional sheet with standard advice on skin care was provided.

At any time that the patient felt stinging, burning, erythema, itching, or discomfort, discontinuation of application was advised. These patients were automatically excluded from the study.

\section{Outcome Assessment}

Complete history and dermatologic evaluation of the participants were done at baseline using a standardized data collection form. Baseline photographs of the test areas were also taken following standard medical photography guidelines. The participants were instructed to return for follow-up after 2 weeks for ODSS, QOL, Corneometer, and Sebumeter readings and follow-up photos as primary outcome measures and to check for patient's assessment of efficacy and the occurrence of adverse events as secondary outcomes.

\section{ODSS}

Based on the European Group on Efficacy Measurement of Cosmetics and other Topical products (EEMCO) guidance for the assessment of xerotic skin conditions, participants were graded by the assessor as follows: 0- absent; 1-fine scaling, faint roughness, and dull; 2- small scales with few large scales, slight roughness, whitish appearance; and 3- small \& large scales uniformly distributed, with definite roughness, slight redness, and few superficial cracks. A decrease of at least 1 point will show improvement.

\section{Dermatology Life Quality Index (DLQI)}

We used the DLQI questionnaire lifted from the "Impact of skin disease on the quality of life of patients", a validated Filipino version ${ }^{11}$ of the Dermatology Life Quality Index (DLQI) questionnaire, a generic skin disease quality of life tool. ${ }^{12}$ The higher the quality of life score, the more quality of life is impaired. Thus, a decrease in the DLQI score would mean improvement in their quality.

\section{Skin hydration and skin lipid measurements}

The corneometer and sebumeter are the most widely used devices to measure skin hydration and skin lipid, and have good accuracy and reproducibility. Measurements were taken at baseline using the Corneometer and Sebumeter probes of the Multi Probe Adapter (MPA) (Courage + Khazaka electronics GmbH Germany). The measurements were taken from the anterior legs immediately below the tibial tuberosity in a well-ventilated area at room temperature. Three measurements were taken and the mean value per participant was recorded. An increase in the measurements from baseline will signify improvement in skin hydration and lipid content. Transepidermal Water Loss (TEWL) on treatment areas should ideally be measured to check for the occlusive effect but this was not performed due to malfunction of the device during the conduct of the study.

\section{Patient's assessment of efficacy}

We asked the patient to assess the efficacy of the study medication on follow -up visit at week 2 using the following rating scale: 0 - not effective; 1 - slightly effective; 2 moderately effective; 3 - markedly effective. Only those who reported moderate to marked effectiveness were considered treatment success.

\section{Adverse events}

During the follow-up visit, the patients were asked about the occurrence of stinging, burning, erythema, itching, and discomfort on the treated skin during the past 2 weeks. Patients were assessed for erythema or other pathologic changes including their severity on the treated skin. Occurrence of any change warranted discontinuance of treatment as stated in their home instruction sheet and automatic exclusion from the study. 


\section{Therapeutic Response}

The investigator reviewed the clinical signs and symptoms at the end of 2-week therapy. The investigator determined the clinical outcome for each participant as follows:

i. 'Treatment Success'-if all three conditions were satisfied:

a. Total clearance or reduction of at least 1 point on the investigator's clinical assessment using the ODSS.

b. Moderate to marked effectiveness based on the Patient's Assessment of Efficacy.

c. Increased skin hydration as measured by Corneometer and increased skin lipids as measured by Sebumeter.

ii. 'Treatment Failure'- if any of the conditions below were observed:

a. No change or increased ODSS from baseline based on clinical assessment.

b. No effectiveness, or only minimal effectiveness based on Patient's Assessment of Efficacy

c. No change or decreasing sebumeter and corneometer measurements.

\section{Data Analysis}

Two types of efficacy analysis were performed: an intention-to-treat analysis (i.e. included the randomized subjects with at least one post-baseline measurement) and combined available case analysis and per protocol analysis (i.e., only compliant patients with complete follow-up visits). The intent to treat population (ITT) included all randomized participants who received at least one application of study medication and who had at least one outcome measure postbaseline within the 14-day study period.

Continuous outcomes such as ODSS, corneometer, and sebumeter measurements were expressed as means and standard deviations. The mean change from baseline measurements was compared between the two groups using Student $\mathrm{t}$-test for unpaired samples. Level of significance was set at $\mathrm{p}<0.05$.

Dichotomous outcomes as to the over-all treatment response were reported as number and percentage of patients. Treatment effects for the primary outcomes (i.e. Clinical endpoint: Treatment failure at 2 weeks) were computed: RRR, ARR, NNT with 95\% confidence intervals. An online
Evidence Based Medicine (EBM) calculator (Centre of EBM Oxford) was used to compute treatment effects.

Adverse events (AE) were categorized and presented as a frequency distribution. Counting of $\mathrm{AE}$ was based upon the number of participants, not the number of adverse events. Participants reporting more than one $\mathrm{AE}$ in an organ system were counted only once in the organ system total.

Missing data may be due to dropouts or withdrawals. Sensitivity analysis using a worst-case scenario was performed to assess the effect of these dropouts and withdrawals on the conclusion of the study. We assumed that dropouts in the VCO group were treatment failures while dropouts in the mineral oil group achieved treatment success.

\section{RESULTS}

\section{Study population}

A total of 188 participants were screened, 148 were randomized (81 for VCO, 67 for mineral oil), and 125 completed the study (74 for VCO, 49 for mineral oil). There were 7 dropouts for VCO and 18 for mineral oil (Figure 1). All participants who followed-up reported good compliance with all instructions.

Fifty-nine (39.8\%) were males and 89 (60.2\%) were females with mean age of 68 years (SD 6.02). The baseline characteristics of the study population are summarized in Table 1.

\section{Investigator's Assessment of Efficacy: Over-all Dry Skin Score}

The distribution of the patients' over-all dry skin score after treatment with virgin coconut oil and mineral oil is presented in Table 2. At baseline, most of the patients in each group had a score of 1 which means that fine scaling, dull and faint roughness were observed in the skin. Two weeks after application of treatments, more than half of the patients per group still had an over-all dry skin score of 1 . However, a trend towards improvement was noted: $43 \%$ (32/74) in the VCO group had no visible signs of leg xerosis versus $22.4 \%(11 / 49)$ in the mineral oil group.

After a 2-week topical application of VCO, 74\% (60/81) of patients achieved success while 34\% (23/67) achieved this in the mineral oil group $(p<0.0001)$ (Table 3). Treatment

Table 1. Baseline characterictics of participants

\begin{tabular}{|c|c|c|c|c|}
\hline Parameter & \multicolumn{2}{|c|}{ VCO Group ( $n=81)$} & \multicolumn{2}{|c|}{ Mineral Oil Group ( $n=67)$} \\
\hline Total number of participants who completed the study & \multicolumn{2}{|c|}{$74(91.4 \%)$} & \multicolumn{2}{|c|}{$49(73.1 \%)$} \\
\hline Mean (SD) Age (years) & \multicolumn{2}{|c|}{68.95 (SD: 7.04 ) } & \multicolumn{2}{|c|}{68.81 (SD: 6.73) } \\
\hline Gender Distribution M/F (\%) & $M=34.6 \%$ & $F=65.4 \%$ & $M=46.3 \%$ & $F=53.7 \%$ \\
\hline Baseline ODSS (mean and standard deviation) & 1 & SD:0.74 & 1 & SD:0.76 \\
\hline Baseline Corneometer (mean and SD) & 66.1 & SD: 58.12 & 60.7 & SD: 27.94 \\
\hline Baseline Sebumeter (mean and SD) & 7.9 & SD: 24.14 & 11.3 & SD: 16.61 \\
\hline Baseline QOL Score (mean and SD) & 13.3 & SD: 1.09 & 13.6 & SD: 1.31 \\
\hline
\end{tabular}




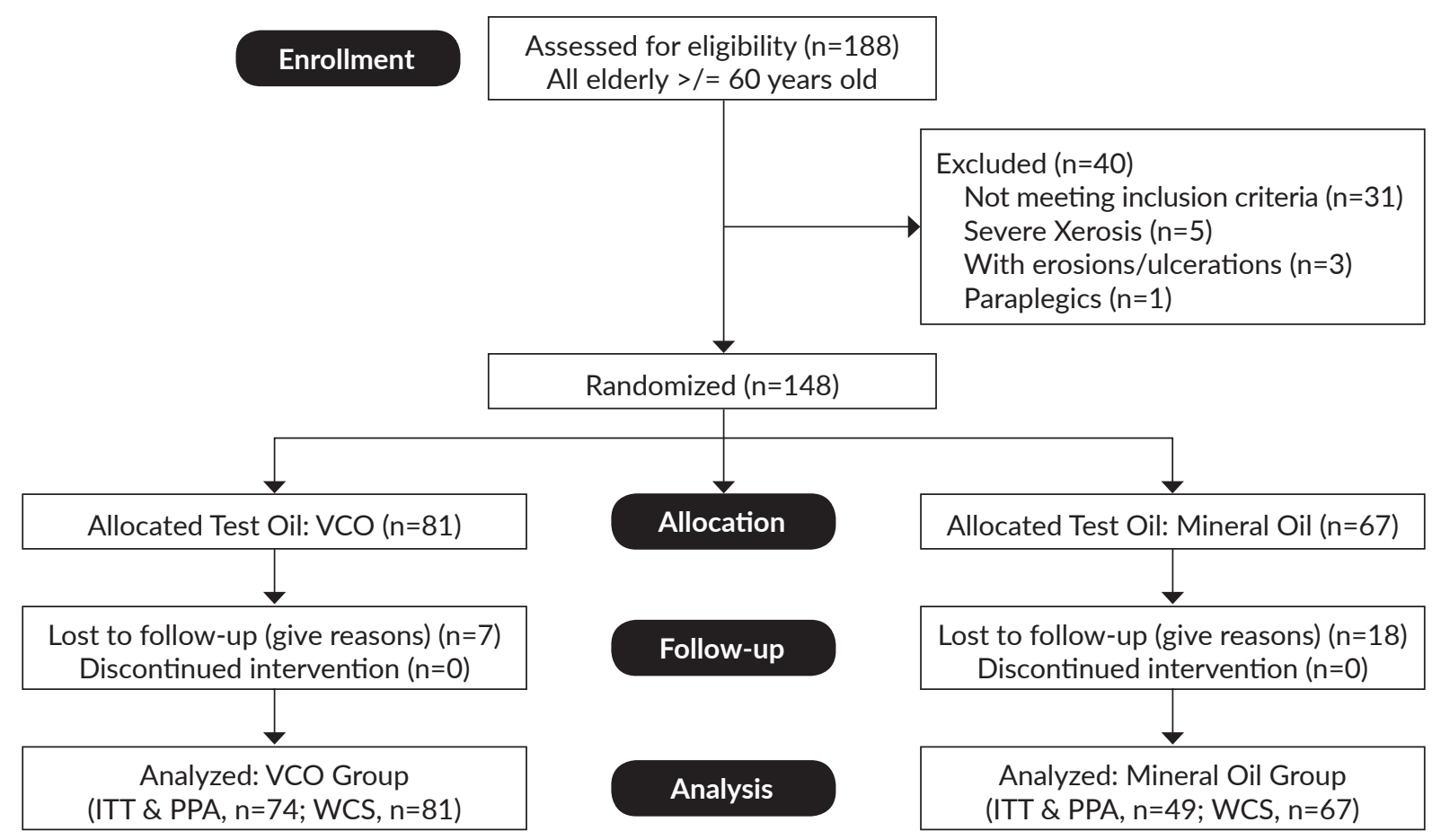

Figure 1. Flow diagram.

Table 2. Distribution of patients according to over-all dry skin score at baseline and post-treatment

\begin{tabular}{|c|c|c|c|c|c|c|c|c|}
\hline \multirow{2}{*}{ ODSS } & \multicolumn{3}{|c|}{ Virgin Coconut Oil } & \multirow{2}{*}{$\%$} & \multicolumn{3}{|c|}{ Mineral Oil } & \multirow{2}{*}{$\%$} \\
\hline & Baseline & $\%$ & Post Tx & & Baseline & $\%$ & Post Tx & \\
\hline 0 & 0 & 0 & 32 & 43.2 & 0 & 0 & 11 & 22.4 \\
\hline 1 & 49 & 60.5 & 40 & 54.1 & 45 & 67.2 & 30 & 61.2 \\
\hline 2 & 26 & 32.1 & 2 & 2.7 & 15 & 22.4 & 8 & 16.3 \\
\hline 3 & 6 & 7.4 & 0 & 0.0 & 7 & 10.4 & 0 & 0.0 \\
\hline Total & 81 & 100.0 & 74 & 100.0 & 67 & 100 & 49 & 100.0 \\
\hline
\end{tabular}

effects computed (based on ITT analysis) using the endpoint of Treatment failure at 2 weeks showed that $\mathrm{VCO}$ reduced the risk of failure compared to mineral oil ( $\mathrm{RRR}=60.5 \%, 95 \%$ CI: 40.7, 73.7). The absolute risk difference was 39.7\% (CI: $2,4)$ favoring VCO treatment. We would need to treat three patients with VCO for 2 weeks to achieve one additional case of success (NNT=3, 95\%) (Table 3 and Table 4A).

Available case analysis of treatment effects as to the ODSS showed that VCO reduced the risk of failure by $64.3 \%$ compared to mineral oil ( $\mathrm{RRR}=64.3 \%, 95 \% \mathrm{CI}: 38.8,79.2)$. Absolute risk difference was 34.1\% (CI: 2, 6). We would need to treat three patients with $\mathrm{VCO}$ for 2 weeks to achieve one additional case of success (NNT $=3,95 \%$ ) (Table 3 and Table 4B).

\section{Quality of Life Measurements}

Baseline DLQI scores were high in both groups, indicating that leg xerosis was a significant problem for the participants. Two weeks after application of the test oils, there was no significant difference in the assessed quality of life of the patients between the two treatments $(p=0.0161)$.
ITT analysis showed that $\mathrm{VCO}$ reduced the risk of failure by $17.3 \%(\mathrm{RRR}=17.3 \%, 95 \% \mathrm{CI}:-16.1,41.1)$. The risk difference was $9 \%(-7.1,25.1)$ favoring VCO. It would take four patients treated with VCO for 2 weeks to have one patient experience moderate to marked efficacy in terms of the patients' QOL measure (NNT $=12,95 \% \mathrm{CI}$ : NNH 4, NNT 14) (Table 3 and Table 4A).

Per protocol analysis of treatment effects as to QOL demonstrated that $\mathrm{VCO}$ reduced the risk of failure by 9.1\% (RRR=9.1\%, 95\% CI: $-32.7,76.7)$. The risk difference between the 2 treatments was $3.1 \%$ (CI: $-14.2,20.5)$ favoring VCO. NNT was 34 (NNT=34, 95\% CI: NNH 5, NNT 7) (Table 3 and Table 4B).

\section{Objective Assessment of Efficacy: Corneometer reading of skin hydration}

Post-treatment, there was a significant difference in the change in mean corneometer measurements between VCO and mineral oil groups. $\mathrm{VCO}$ showed significantly greater skin hydration at $74 \%(60 / 81)$ as compared to $46 \%(31 / 67)$ in the mineral oil group $(p<0.001)$ (Figure 2$)$. 


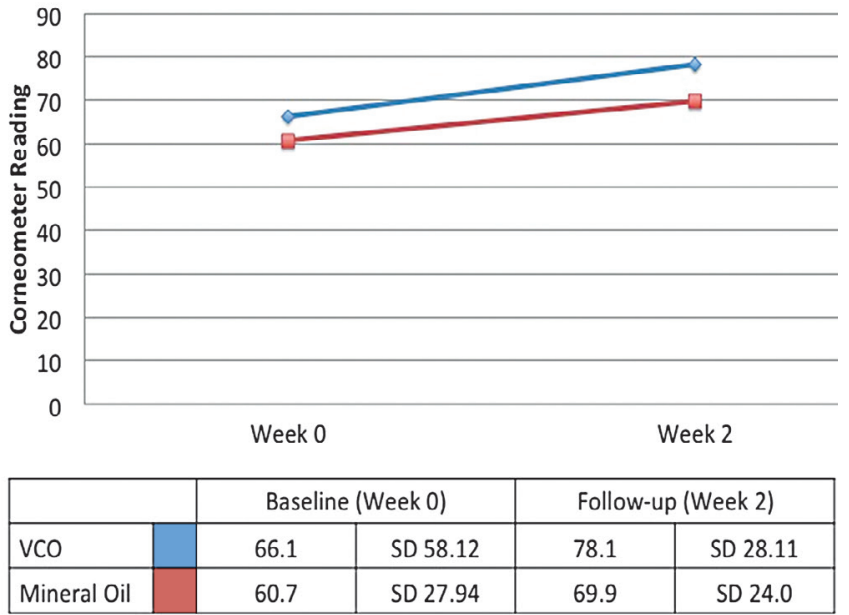

Figure 2. Corneometer values at baseline and 2 weeks post treatment.

Table 3. Summary of therapeutic response rates, by parameter (ITT \& PPA analysis)

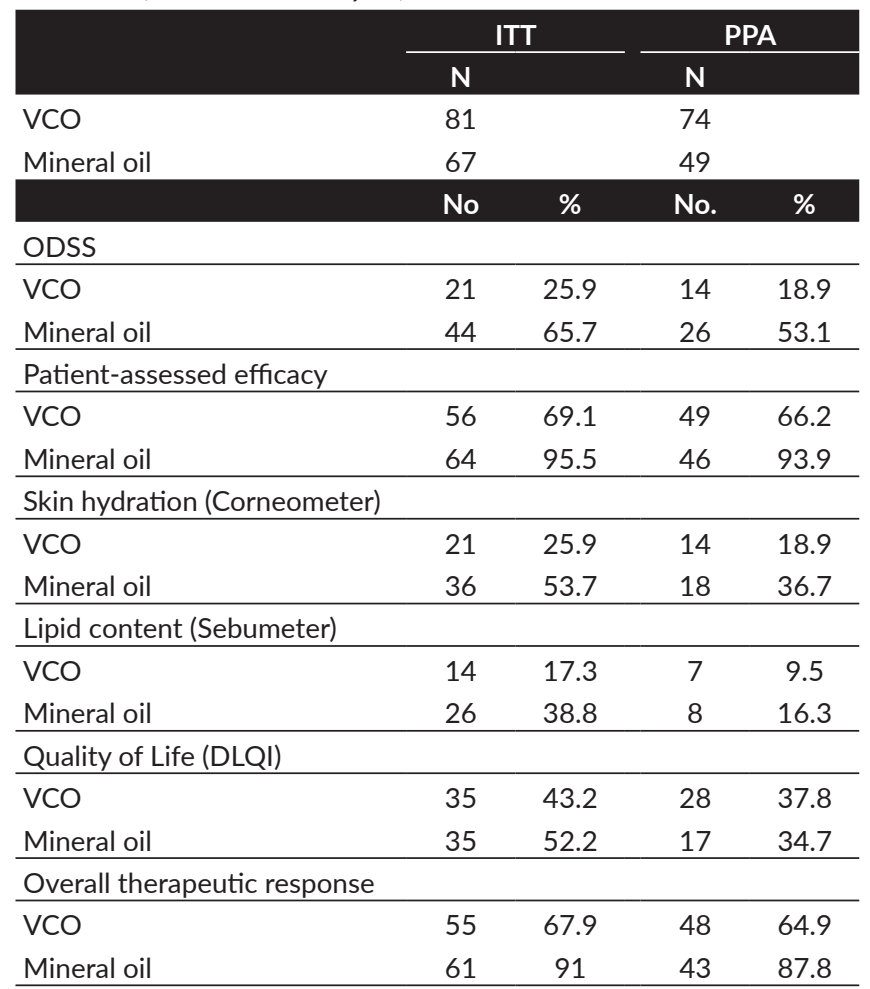

On ITT analysis, VCO reduced failure based on corneometer readings by $51.7 \%(\mathrm{RRR}=51.7 \%, 95 \% \mathrm{CI}: 25.8$ to 68.6) compared to mineral oil. VCO reduced the risk of treatment failure by $27.8 \%(\mathrm{ARR}=27.8 \%, 95 \% \mathrm{CI}: 12.5$, 43.1). NNT was 4 (NNT $=4,95 \%$ CI: 2-8) (Table 3 and Table 4).

Per protocol analysis showed that VCO reduced the risk of failure by $48.5 \%(\mathrm{RRR}=48.5 \%, 95 \% \mathrm{CI}$ : $6.4,71.7)$ compared to mineral oil. The absolute reduction in the risk
(ARR) of treatment failure was $17.8 \%$ (95\% CI: 1.6, 34). It would take 6 patients treated with VCO for 2 weeks to have one additional patient with treatment success based on corneometer readings (NNT=6, 95\% CI: 3, 62) (Table 3 and Table 4B).

\section{Objective Assessment of Efficacy: Sebumeter reading of skin lipid}

After 2 weeks of treatment, there was no significant difference in the mean sebumeter measurements between patients who applied VCO and mineral oil. Improvement in the skin lipid contents was observed using the sebumeter with $82.7 \%(67 / 81)$ in the VCO group compared to $61.2 \%$ $(41 / 67)$ in the mineral oil group $(p=0.6591)$.

ITT analysis showed VCO to reduce the risk of failure based on sebumeter readings by $55.5 \%$ ( $R R R=55.5 \%, 95 \%$ CI: 21.8, 74.6) while the absolute risk reduction (ARR) between treatments was only $21.5 \%$, favoring VCO (95\% CI: 7.2, 35.8). It would take five patients treated with VCO for 2 weeks to have one additional patient with treatment success based on sebumeter readings (NNT $=5,95 \% \mathrm{CI}: 3,14$ ) (Table 3 and Table 4A).

Per protocol analysis on the other hand showed VCO reduced the risk of failure based on sebumeter readings by 42.1\%（RRR=42.1\%, 95\% CI: $-49.5,77.5)$ compared to mineral oil. VCO reduced the risk of treatment failure by 6.9\% (ARR $=6.9 \%, 95 \%$ CI: $-5.4,19.2)$. NNT was $15(95 \%$ CI: NNH 5, NNT 19) (Table 3 and Table 4B).

\section{Patient's Assessment of Efficacy}

Majority (7 out of 10) of participants found the oil assigned to them as slightly effective (efficacy score=1) (Table 5). Moreover, the patients' assessment of efficacy of the oil applied on their skin showed a significantly higher percentage $29.6 \%(24 / 81)$ in the VCO group compared to $5.9 \%(4 / 67)$ in the mineral oil group $\left(C h i^{2}=8.895 ; p=0.003\right)$.

ITT analysis of treatment effects as to patients' assessment of efficacy using the endpoint of treatment failure at 2 weeks showed that VCO reduced the risk of failure by $27.6 \%(\mathrm{RRR}=27.6 \%, 95 \% \mathrm{CI}: 15.5,38)$. The risk difference between the 2 treatments was $26.4 \%$ favoring VCO. NNT was 4 (95\% CI: 3-7) (Table 3 and 3A).

Per protocol analysis showed that VCO reduced the risk of failure by $29.5 \%(\mathrm{RRR}=29.5 \%, 95 \% \mathrm{CI}: 15.7,41)$. The risk difference between the 2 treatments was $27.7 \%$ favoring VCO. It would take four patients treated with VCO for 2 weeks to have one additional patient with moderate to marked efficacy in terms of the patients' self-assessment (NNT $=4,95 \%$ CI: 2, 7) (Table 3 and 4B).

\section{Over-all Therapeutic Response}

In the VCO group, 32.1\% (26/81) achieved treatment success compared to only $8.9 \%(6 / 67)$ in the mineral oil group $(p=0.004614)$. ITT analysis based on over-all therapeutic response showed VCO to reduce the risk of 
Table 4A. Summary of treatment effects: VCO versus mineral oil at week 2 (ITT analysis)

\begin{tabular}{|c|c|c|c|c|c|c|}
\hline Parameter & RRR (\%) & $95 \% \mathrm{Cl}$ & ARR (\%) & $95 \% \mathrm{Cl}$ & NNT & $95 \% \mathrm{Cl}$ \\
\hline ODSS & 60.5 & $40.7,73.7$ & 39.7 & $24.9,54.6$ & 3 & 2,4 \\
\hline Patient Assessment & 27.6 & $15.5,38$ & 26.4 & $15.2,37.6$ & 4 & 3,7 \\
\hline Skin hydration (Corneometer) & 51.7 & $25.8,68.6$ & 27.8 & $12.5,43.1$ & 4 & 2,8 \\
\hline Lipid content (Sebumeter) & 55.5 & $21.8,74.6$ & 21.5 & $7.2,35.8$ & 5 & 3,14 \\
\hline Quality of Life & 17.3 & $-16.1,41.1$ & 9 & $-7.1,25.1$ & 12 & NNH 4, NNT 14 \\
\hline Over-all Therapeutic Response & 25.4 & $11.8,36.9$ & 23.1 & $10.9,35.4$ & 5 & 3,9 \\
\hline
\end{tabular}

Table 4B. Summary of treatment effects: VCO versus mineral oil at week 2 (per protocol analysis)

\begin{tabular}{ccccccc} 
Parameter & RRR (\%) & $\mathbf{9 5 \% ~ C l}$ & ARR (\%) & $\mathbf{9 5 \% ~ C l}$ & NNT & 95\% Cl \\
ODSS & 64.3 & $38.8,79.2$ & 34.1 & $17.6,50.7$ & 3 & 2,6 \\
Patient Assessment & 29.5 & $15.7,41$ & 27.7 & $15,40.4$ & 4 & 2,7 \\
Skin hydration (Corneometer) & 48.5 & $6.4,71.7$ & 17.8 & $1.6,34$ & 6 & 3,62 \\
Lipid content (Sebumeter) & 42.1 & $-49.5,77.5$ & 6.9 & $-5.4,19.2$ & 15 & NNH 5, NNT 19 \\
Quality of Life & 9.1 & $-32.7,76.7$ & 3.1 & $-14.2,20.5$ & 32 & NNH 5, NNT 7 \\
Over-all Therapeutic Response & 26.1 & $9.9,39.3$ & 22.9 & $8.7,37.1$ & 5 & 3,11 \\
\hline
\end{tabular}

Table 5. Distribution of patients according to perceived efficacy of the study medication after two weeks follow-up visit

\begin{tabular}{|c|c|c|c|c|}
\hline \multirow{3}{*}{ Patient's Assessment of Efficacy } & \multicolumn{4}{|c|}{ Treatment } \\
\hline & \multicolumn{2}{|c|}{ Virgin Coconut Oil ( $n=70)$} & \multicolumn{2}{|c|}{ Mineral Oil $(n=47)$} \\
\hline & 4 & $5.7 \%$ & 5 & $10.6 \%$ \\
\hline Slightly effective & 42 & $60 \%$ & 38 & $80.8 \%$ \\
\hline Moderately effective & 24 & $34.3 \%$ & 4 & $8.5 \%$ \\
\hline
\end{tabular}

Table 6. Over-all therapeutic response of VCO vs. mineral oil based at week 2 (sensitivity analysis)

\begin{tabular}{|c|c|c|c|c|c|c|c|}
\hline $\begin{array}{l}\text { VCO } \\
\mathrm{N}=81\end{array}$ & $\begin{array}{l}\text { Mineral Oil } \\
\qquad N=67\end{array}$ & RRI & $95 \% \mathrm{Cl}$ & ARI & $95 \% \mathrm{Cl}$ & NNT & $95 \% \mathrm{Cl}$ \\
\hline $67.9 \%$ & $64.2 \%$ & $5.8 \%$ & $-16.2,33.6 \%$ & $3.7 \%$ & $-11.6,19.1 \%$ & 27 & NNH 3, NNT 11 \\
\hline
\end{tabular}

failure by $25.4 \%(\mathrm{RRR}=25.4 \%, 95 \% \mathrm{CI}: 11.8,36.9)$ with a risk difference between treatments of $23.1 \%$, favoring $\mathrm{VCO}$ (95\% CI: 10.9, 35.4).It would take five patients treated with $\mathrm{VCO}$ for 2 weeks to have one additional patient to experience reduction of xerosis of the legs (NNT $=5,95 \%$ CI: 3, 9).

Per protocol analysis showed that $\mathrm{VCO}$ reduced the risk of failure by $26.1 \%(\mathrm{RRR}=26.1 \%, 95 \% \mathrm{CI}: 9.9,39.3)$ compared to mineral oil. VCO reduced the risk of treatment failure by $22.9 \%(A R R=22.9 \%, 95 \% \mathrm{CI}: 8.7,37.1)$. NNT was 5 (NNT $=5,95 \% \mathrm{CI}: 3,11)$. These point estimates were all statistically significant (Table 5 ).

\section{Sensitivity Analysis}

Worst-case scenario sensitivity analysis was performed to determine the effect of dropout rates on the efficacy rates. The VCO group had 7 dropouts (8.6\%) while the mineral oil group had 18 drop outs (26.9\%), considered a worrisome dropout rate. We assumed that dropouts in the VCO group were treatment failures; while dropouts in mineral oil groups achieves treatment success.

The treatment effects showed a trend towards harm for the VCO treatment with 55/81 (67.9\%) compared to
$43 / 67(64.2 \%)$ in the mineral oil group, however these were not statistically significant. The conclusion regarding $\mathrm{VCO}$ treatment changes when the effect of the dropouts is considered in the analysis (Table 6).

\section{Adverse Events}

Eight percent (6/74) of patients in the VCO group and $26.5 \%(13 / 49)$ in the mineral oil group experienced adverse effects (erythema, pruritus, and discomfort). These were however mild and transient and the patients were able to tolerate and complete the treatment. The difference in the occurrence of adverse effects between the two groups was not statistically significant $(p=0.089)$.

\section{DISCUSSION}

Over-all, this study was able to show the superior efficacy of VCO over mineral oil as a therapeutic moisturizer for mild to moderate senile xerosis of the legs. The results of our study were consistent with a previous double-blind RCT, which compared EVCO versus mineral oil on mild to moderate xerosis in 34 Filipino adult patients. Both groups showed significant improvement in skin hydration 
and surface lipid levels. Subjective grading from both the investigators and the participants showed a general trend favoring EVCO in perceived improvement, better absorption into dry skin, easier spreading, better feel upon and after application, and an acceptable odor. ${ }^{13}$ Our study, on the other hand, revealed that there was a significant difference between the patient's assessment of efficacy of the two treatments, favoring VCO. Although an attempt to blind the participants of their assigned test oil was made, the characteristic aroma and physical nature of $\mathrm{VCO}$ would easily be noticed; this prevented complete blinding of the participants. But with the consistent results of both objective assessment and the participants' subjective assessment, we can assume that identification of VCO, as the test oil, did not significantly affect the outcome of this study.

The subjective and objective measures of efficacy were also consistent with other studies on the effects of $\mathrm{VCO}$ on chronic xerotic skin conditions like atopic dermatitis. In a double-blind RCT, among pediatric patients with mild to moderate atopic dermatitis, topical application of VCO for eight weeks was superior to that of mineral oil based on clinical (SCORAD) and instrumental (TEWL, skin capacitance) assessments. ${ }^{14}$ Mean SCORAD indices decreased from baseline by $68.23 \%$ in the VCO group and by $38.13 \%$ in the mineral oil group $(p<0.001)$. The VCO group achieved a post-treatment mean TEWL of 7.09 from a baseline mean of 26.68 , whereas the mineral oil group demonstrated baseline and post-treatment TEWL values of 24.12 and 13.55 , respectively. In the VCO group, posttreatment skin capacitance rose to 42.3 from a baseline mean of 32.0, whereas that in the mineral oil group increased to 37.49 from a baseline mean of 31.31. In addition, another RCT on adult atopic dermatitis patients published in 2008 showed beneficial effects of VCO versus virgin olive oil (VOO) in Dry Skin Score reduction and in in vitro broadspectrum activity against Staphylococcus aureus, fungi, and viruses. In the objective-SCORAD Severity Index, the difference was not significant at baseline $(\mathrm{p}=0.15)$ but was significantly different post treatment $(\mathrm{p}=0.004)$; this was reduced for both oils $(p<0.005)$ but was greater with VCO. Of the 20 patients whose cultures were positive and who were randomized to $\mathrm{VCO}, 1$ participant (5\%) remained positive; of the 12 patients whose cultures were positive and who were randomized to the VOO arm, 6 (50\%) remained positive. Compared to the $\mathrm{VOO}$ arm, the calculated relative risk of nontreatment was $0.10 \mathrm{among}$ those in the $\mathrm{VCO}$ arm and was thus superior to that of the control arm. Hence, VCO maybe useful in the proactive treatment of adult atopic dermatitis colonization. ${ }^{15}$

$\mathrm{VCO}$ acts as an effective replacement for lost lipids in xerotic skin. These natural fatty acids are not found in mineral oil. Our study showed no significant difference in the mean sebumeter measurements between patients who applied VCO and mineral oil after 2 weeks. But when any improvement in skin lipid content was used as a clinical endpoint, $\mathrm{VCO}$ was significantly superior to mineral oil. The QOL scores in our study improved from baseline with both study oils but did not differ significantly between treatments. This may be due to the mild nature of the xerosis of some of the participants hence not causing much difference in their QOL scores. The low NNT of VCO treatment indicate that the cost of xerosis treatment will also be relatively low compared to mineral oil treatment. Using the NNT for the over-all efficacy and computation for the cost of treatment in order to achieve improvement or clearance of leg xerosis in one patient, $\mathrm{VCO}$ treatment would cost an average of P125 to achieve one treatment success after 2 weeks of use. VCO treatment costs can range from as low as $\mathrm{P} 75$ to as high as $\mathrm{P} 225$ to achieve one treatment success by the end of 2 weeks.

Although our study met the target sample size, a widerange in the confidence intervals indicated lack of precision in the estimates of some effects. The investigators tried to ensure good compliance in the proper application of the test oils and measured this among the participants, however, an imbalance in the treatment allocation and high dropout rates was still observed. Participants were randomized using a computer-generated list in balanced blocks of 10 according to the calculated sample size of only 144 . However, during the course of the study, with 188 subjects qualified, all subjects who were not initially included in the computerized allocation were randomized through toss coin. This has caused unequal allocation favoring the experimental oil, VCO.

The investigators tried to ensure retention of the participants by providing compensation and thorough explanation of the importance of the study. However, there were 7 drop-outs $(8.6 \%)$ in the VCO group while the mineral group had 18 drop outs (26.9\%) which was considered a worrisome drop-out rate. These patients were lost to follow-up. While some dropouts will always be inevitable, we can only presume other reasons for dropping out like conflicts in schedule, inconvenience, physical constraints, etc. To address this concern, sensitivity analysis using the extreme case scenario was performed to determine the effect of the dropout rates on efficacy rates; this showed no statistical significance.

Our study only measured the immediate or short-term moisturizing effects of $\mathrm{VCO}$. A longer treatment period will be necessary to measure the long-term efficacy of VCO as a moisturizer.

\section{CONCLUSION}

This study validated the use of an indigenous agricultural product, virgin coconut oil, in the treatment of senile xerosis. VCO is a locally produced, culturally acceptable, and traditionally used remedy. It is potentially a cost-effective treatment for mild to moderate xerosis among the elderly population but a health economic evaluation study has to be 
conducted to confirm this. The more widespread use of VCO here and abroad will further promote the Philippine coconut industry and will potentially contribute to the improvement of our economy. Since VCO is locally produced, patients will have better access to this moisturizing oil than the imported mineral oil.

Among elderly patients with mild to moderate leg xerosis, topical VCO application for 2 weeks was superior to mineral oil in the immediate improvement of leg xerosis based on the primary outcome of measures (ODSS, QOL, and Corneometer and Sebumeter readings) for treatment success.

\section{Statement of Authorship}

All authors approved the final version submitted.

\section{Author Disclosure}

All authors declared no conflict of interest.

\section{Funding Source}

None.

\section{REFERENCES}

1. Reszke R, Pelka D, Walasek A, Machaj Z, Reich A. Skin disorders in elderly subjects. Int J Dermatol. 2015 Sep; 54 (9):e332-8.

2. Sheshala R, Ying LT, Hui LS, Barua A, Dua K. Development and Anti-microbial Potential of Topical Formulations Containing Cocos nucifera Linn. Antiinflamm Antiallergy Agents Med Chem. 2013; 12 (3):253-64.

3. Nevin KG, Rajamohan T. Effect of topical application of virgin coconut oil on skin components and antioxidant status during dermal wound healing in young rats. Skin Pharmacol Physiol. 2010; 23 (6):290-7.

4. Purnamawati S, Indrastuit N, Danarti, R, Saefudin T. The Role of Moisturizers in Addressing Various Kinds of Dermatitis: A Review. Clin Med Res. 2017 Dec; 15 (3-4):75-87.
5. Kabara JJ. Toxicological, bacteriocidal and fungicidal properties of fatty acids and some derivatives. J Am Oil Chem Soc. 1979 Nov; 56 (11):760A-767A.

6. Abraham RL, Verallo-Rowell VM. Safety and efficacy of monolaurin, a coconut oil extract, versus ethyl alcohol in rinse- free hand antiseptic gels on healthcare personnel's hands and microbial isolates. J Phil Dermatol Soc. 2001 Nov; 10 (2):90-9.

7. Dim-Jamora KCC, Verallo-Rowell VM. A double-blind RCT comparing 2\% monolaurin cream and 2\% Mupirocin cream in the treatment of superficial bacterial skin infections. J Phil Dermatol Soc. 2006.15; 2:31-6.

8. Rosado A, Fernandez-Rivas M, Gonzalez-Mancebo E, Leon F, Campos C, Tejedor MA. Anaphylaxis to coconut. Allergy. 2002 Feb; $57(2): 182-3$.

9. Burnett CL, Bergfeld WF, Belsito DV, et al. Final report on the safety assessment of Cocos nucifera (coconut) oil and related ingredients. Int J Toxicol. 2011 May; 30 (3 Suppl):5S-16S.

10. Organic Virgin Coconut Oil, Specialty coconut product line [Internet]. [cited 2017 Oct]. Available from: https://www.peterpaul.com.ph/ products/specialtycoconut productline/organicvirgincoconutoil.

11. Santos M, Balagat R, Frez L. The Impact of Skin Disease on the Quality of Life of patients at the Philippine General Hospital Outpatient Clinic. J Phil Dermatol Soc. 2005 Nov; 14 (2):9-1

12. Finlay AY, Khan GK. Dermatology Life Quality Index (DLQI)--a simple practical measure for routine clinical use. Clin Exp Dermatol. 1994 May; 19 (3): 210-6

13. Agero AL, Verallo-Rowell VM. A randomized double-blind controlled trial comparing extra virgin coconut oil with mineral oil as a moisturizer for mild to moderate xerosis. Dermatitis. 2004 Sep; 15 (3):109-16

14. Evangelista MT, Abad-Casintahan F, Lopez-Villafuerte L. The effect of topical virgin coconut oil on SCORAD index, transepidermal water loss, and skin capacitance in mild to moderate pediatric atopic dermatitis: a randomized, double-blind, clinical trial. Int J Dermatol. 2014 Jan; 53 (1):100-8

15. Verallo-Rowell VM, Dillague KM, Syah-Tjundawan BS. Novel antibacterial and emollient effects of coconut and virgin olive oils in adult atopic dermatitis. Dermatitis. 2008 Nov-Dec; 19 (6):308-15. 